\title{
Long-Term Clinical Outcomes of Unprotected Left Main Percutaneous Coronary Intervention: A Large Single-Centre Experience
}

\author{
Lijian Gao (i), ${ }^{1}$ Zhan Gao, ${ }^{1}$ Ying Song, ${ }^{1}$ Changdong Guan, ${ }^{2}$ Bo Xu, ${ }^{2}$ Jue Chen, ${ }^{1}$ Haibo Liu, ${ }^{1}$ \\ Xuewen Qin, ${ }^{1}$ Min Yao, ${ }^{1}$ Jinqing Yuan, ${ }^{1}$ Yongjian $\mathrm{Wu},{ }^{1}$ Fenghuan $\mathrm{Hu},{ }^{1}$ Jie Qian, ${ }^{1}$ \\ Yida Tang, ${ }^{1}$ Kefei Dou, ${ }^{1}$ Weixian Yang, ${ }^{1}$ Hong Qiu, ${ }^{1}$ Chaowei Mu, ${ }^{1}$ Jun Dai, ${ }^{1}$ Shubin Qiao, ${ }^{1}$ \\ Jilin Chen, ${ }^{1}$ Runlin Gao, ${ }^{1}$ and Yuejin Yang (DD $^{1}$ \\ ${ }^{1}$ Department of Cardiology, Coronary Heart Disease Center, Fuwai Hospital, National Center for Cardiovascular Diseases, \\ Chinese Academy of Medical Science and Peking Union Medical College, Beijing, China \\ ${ }^{2}$ Catheterization Laboratories, Fu Wai Hospital, National Center for Cardiovascular Diseases, \\ Chinese Academy of Medical Sciences, Beijing, China
}

Correspondence should be addressed to Yuejin Yang; yangyjfw@126.com

Received 26 July 2020; Revised 16 December 2020; Accepted 30 December 2020; Published 12 January 2021

Academic Editor: Seif S. El-Jack

Copyright (c) 2021 Lijian Gao et al. This is an open access article distributed under the Creative Commons Attribution License, which permits unrestricted use, distribution, and reproduction in any medium, provided the original work is properly cited.

\begin{abstract}
Aims. This study sought to report the 10-year clinical outcomes of patients who underwent unprotected left main (LM) percutaneous coronary intervention (PCI) in a large centre. Methods and Results. A total of 913 consecutive patients who underwent unprotected LM PCI from January 2004 to December 2008 at Fu Wai Hospital were retrospectively analysed; the mean age was $60.0 \pm 10.9$ years, females accounted for $22 \%$ of patients, diabetes was present in $27.7 \%$ of patients, and an LM bifurcation lesion occurred in $82.9 \%$ of patients. During the median follow-up of 9.7 years, major adverse cardiac or cerebrovascular events (MACCEs) occurred in $25.6 \%$ (234) of patients, and the rates of all-cause death, myocardial infarction, and stroke were $14.9 \%$, $11.0 \%$, and $7.1 \%$, respectively. Cardiac death occurred in only $7.9 \%$ of patients. The estimated event rate was $41.9 \%$ for death/ myocardial infarction/any revascularization and $45.9 \%$ for death/MI/stroke/any revascularization. Definite/probable stent thrombosis occurred in 4.3\% (39) of patients. According to the subgroup analysis, IVUS-guided PCI was associated with less longterm MACCEs. Further multivariate analysis identified that age and LVEF $<40 \%$ were the only independent predictors for 10 -year death. Age, $\mathrm{LVEF}<40 \%$, creatinine clearance, and incomplete revascularization were independent predictors for death/MI, while a two-stent strategy, diabetes, a transradial approach, and the use of bare metal stents (BMSs) or first-generation drug-eluting stents (DESs) were not. Conclusions. Unprotected LM PCI in a large cohort of consecutive patients in a single large centre demonstrated favourable long-term outcomes up to 10 years even with the use of BMSs and first-generation of DESs.
\end{abstract}

\section{Introduction}

Although randomized controlled trials comparing the effect of percutaneous coronary interventions (PCIs) and coronary artery bypass graft (CABG) still have not reached consistent results in patients with unprotected left main (LM) coronary artery disease (CAD) $[1,2]$, PCI has always been recommended as an effective treatment for patients with unprotected LM CAD by guidelines $[3,4]$ and has been performed in daily practice, except in bifurcated lesion and the twostent strategy [5].

During the last 2 decades, therapeutic advancements including drug-eluting stents (DESs) [6-11] and invasive imaging tools such as intravascular ultrasound (IVUS) [12-17] have largely improved PCI outcomes in patients with unprotected LM disease. In addition, increased experience in complex LM stenting [18] has further improved interventional device-oriented outcomes [19]. However, the 
long-term outcomes of patients undergoing LM PCI have always been a concern due to suboptimal results, such as stent underexpansion or malapposition at this particular part, which make patients prone to stent thrombosis and instent restenosis, leading to devastating consequences, including death [20-22]. Previous reports have revealed more favourable outcomes in LM patients who underwent PCI than in those who underwent CABG or medical therapy alone; however, the long-term follow-up results showed conflicting reversed findings $[23,24]$. On the other hand, clinical or technique factors that influence long-term outcomes following LM are still controversial. In this circumstance, we retrospectively collected over 900 consecutive LM patients with detailed patient demographics, lesion, and procedural information who underwent LM PCI at a large cardiac centre with as long as a 10-year follow-up duration. The study sought to analyse the very long-term performance of PCI for LM disease and investigate potential factors that influence long-term outcomes.

\section{Methods}

2.1. Study Population. Between January 2004 and December 2008, 19,600 patients underwent PCI at Fu Wai Hospital; among them, a total of 916 consecutive patients diagnosed with LM diseases were retrospectively collected. The exclusion criteria were age $<18$ years and missing major baseline information. After excluding 3 patients who met the exclusion criteria, 913 LM PCI patients were finally analysed (Figure 1). Unprotected LM disease was defined as documented myocardial ischaemia with $\geq 50 \%$ LM stenosis and no patent bypass graft to the left anterior descending or left circumflex arteries. The population of patients who were rejected by surgeons from surgery included those who met any 1 of the following criteria: chronic obstructive pulmonary disease, left ventricular ejection fraction $<35 \%$ with minimal or without viable myocardium, age $>70$ years, acute myocardial infarction with haemodynamic instability, creatinine clearance $<50 \mathrm{ml} / \mathrm{min}$, or bleeding history within 6 months. Clinical, procedural, and outcomes data were recorded in a dedicated database. The baseline and residual SYNTAX Score (SS) were assessed using standard quantitative coronary analysis methodology by an independent angiographic core laboratory. Follow-up was performed via an office visit or telephone contact at 30 days and annually thereafter.

2.2. Procedures. All patients undergoing PCI were prescribed aspirin plus clopidogrel (loading dose, 300 or $600 \mathrm{mg}$ ) before the coronary intervention unless they had previously received regular antiplatelet medications. Procedures were performed with standard interventional techniques. Lesions in the ostium or body of the LM usually received a single stent with the postdilation technique; if the single stent crossed over the LM bifurcation to the LAD, postdilatation with kissing balloon angioplasty was used at the operator's discretion to finish the procedure. When treating distal LM bifurcation, the
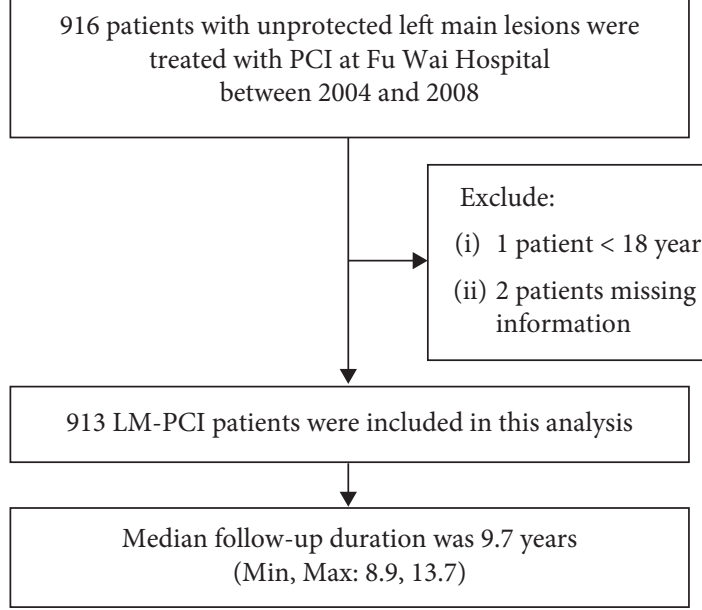

Figure 1: Patient flow. PCI = percutaneous coronary intervention; $\mathrm{LM}=$ left main.

operators decided the strategy of a 1- or 2-stent technique. When the 2-stent strategy was applied, the proximal optimization technique and postdilatation with kissing balloon angioplasty was mandatory to achieve complete apposition of the LM stent. The use of intravascular ultrasound (IVUS) or optical coherence tomography (OCT) was at the operator's discretion, and an intra-aortic balloon bump (IABP) was used as mechanical support in patients with a very low LVEF or other complications. The use of dual antiplatelet therapy with aspirin and clopidogrel was recommended for, at least, 12 months after stent implantation.

2.3. Endpoints and Definitions. The present study evaluated the long-term safety and efficacy following LM PCI. The primary safety endpoint was the composite endpoint of death, MI, and stroke. The primary efficacy endpoint was target-vessel revascularization (TVR). The secondary endpoints included individual components of the composite outcome, cardiac death, any revascularization, target lesion revascularization (TLR), and stent thrombosis as defined according to definite or probable Academic Research Consortium (ARC) criteria [22]. Cardiac death was defined as any death that could not be attributed to a noncardiac cause. Periprocedural MI was defined as a creatine kinase concentration $>2$ times the upper limit of normal within 48 hours after the procedure, and TVR was defined as any revascularization within the entire major coronary vessels proximal or distal to a target lesion, including upstream and downstream side branches and the target lesion itself.

2.4. Statistical Analysis. Continuous variables are presented as the mean \pm SD and were compared by Student's t-test. Categorical variables are presented as percentages and counts; between-group differences were compared by the chi-square test or Fisher's exact test. Subgroup analyses were performed to identify long-term outcome predictors after 
LM PCI among different populations including patients who were suitable for surgery versus the regular population, the transradial versus the transfemoral approach, bifurcation versus nonbifurcation lesions, one- versus two-stent strategy, BMS versus DES treatments, and treatment with or without IVUS guidance. The ten-year outcomes in the overall population and subgroups are presented as Kaplan-Meier estimates and were compared using the logrank test. Multivariable Cox proportional hazards models were constructed to identify independent predictors of 10year all-cause death, cardiac death, and death/MI. All statistical analyses were performed using SAS version 9.1.3 (SAS Institute, Cary, North Carolina).

\section{Results}

3.1. Clinical and Procedural Characteristics. Baseline characteristics are presented in Table 1. A total of 913 LM PCI patients with the mean age of $60.0 \pm 10.9$ years were enrolled; females accounted for $22.0 \%$ of patients, diabetes was present in $27.7 \%$ of patients, and unstable angina was present in $60.4 \%$ of patients. An LM bifurcation lesion was present in $82.9 \%$ of patients, LM plus 3-vessel disease was present in $36.1 \%$ of patients, and the mean LVEF was $62.5 \pm 7.9 \%$. Patients with a SYNTAX score $\leq 32$ and $>32$ accounted for $86.3 \%$ and $13.7 \%$, respectively. LM PCI was performed with a transradial approach in $45.7 \%$ of the patients, the 2-stent strategy in $26.5 \%$, and IVUS guidance in $39.5 \%$, as shown in Table 2.

The median follow-up duration of these patients was 9.7 years (min, max: 8.9, 13.7 years). The composite endpoint of death/MI/stroke occurred in 234 (25.6\%, 95\% confidence interval [CI]: $22.7-28.5 \%)$ patients, the rate of all-cause death was $14.9 \%$ (136), and $11.0 \%(100)$ and $7.1 \%(65)$ of patients suffered MI and stroke, respectively (Table 3 and Figure 2). The 10-year estimated incidence of any revascularization was $25.0 \%$, and the TVR and target lesion revascularization (TLR) were $16.1 \%$ and $9.9 \%$, respectively, with increase rates $1.4 \%$ (TVR) and $0.9 \%$ (TLR) annually (Figure 3). Up to 10 years, 136 (14.9\%) patients died; among them, $72(7.9 \%)$ patients died due to cardiac events (Appendix). Definite/probable stent thrombosis occurred in 39 (4.3\%) patients.

3.2. Subgroup Analyses. A total of 197 (21.6\%) patients in the present study were rejected by surgeons due to patient comorbidities or surgical ineligibility. Kaplan-Meier curves showed that there were no differences between patients who underwent the transradial or transfemoral approach (logrank $p=0.69$ ), those with LM bifurcation or nonbifurcation lesions ( $\log$-rank $p=0.97$ ), or those treated with the twostent or one-stent strategy $(\log$-rank $p=0.28)$, while the 10 year death/MI/stroke rate was significantly higher in patients who were rejected by surgeons $(\log$-rank $p<0.0001)$ and in patients with implantation of BMS $(32.7 \%$ vs. $23.9 \%$, logrank $p=0.04)$. On the other hand, an LM PCI procedure guided by IVUS significantly reduced long-term death/MI/ stroke $(20.8 \%$ vs. $27.7 \%$, log-rank $p=0.03)$ (Figure 4$)$.
TABLE 1: Baseline patient characteristics.

\begin{tabular}{lc}
\hline & $N=913$ \\
\hline Age, years & $60.0 \pm 10.9$ \\
Female & $22.0 \%(201)$ \\
Body mass index, $\mathrm{kg} / \mathrm{m}^{2}$ & $25.6 \pm 3.1$ \\
Diabetes & $27.7 \%(253)$ \\
Insulin requiring & $0.4 \%(4)$ \\
Current smoking & $33.4 \%(305)$ \\
Hypertension & $59.6 \%(544)$ \\
Hyperlipidaemia & $49.8 \%(455)$ \\
Family history of coronary artery disease & $14.5 \%(132)$ \\
Previous percutaneous coronary intervention & $24.1 \%(220)$ \\
Prior myocardial infarction & $30.9 \%(282)$ \\
Prior stroke & $6.9 \%(63)$ \\
Peripheral arterial disease & $3.3 \%(30)$ \\
Unstable angina & $60.4 \%(551)$ \\
LVEF, \% & $62.5 \pm 7.9$ \\
LVEF $<40 \%$ & $1.3 \%(12)$ \\
LVEF $40 \%-50 \%$ & $5.8 \%(53)$ \\
LVEF $>50 \%$ & $92.9 \%(848)$ \\
\hline
\end{tabular}

Values are reported as the mean $\pm \mathrm{SD}$ or $\%(n) . \mathrm{LVEF}=$ left ventricular ejection fraction.

3.3. Multivariate Analysis. In the multivariate analysis, we found that age and a left ventricular eject fraction (LVEF) $<40 \%$ were independent predictors for 10-year death (all $p<0.01)$. In addition, age, $\mathrm{LVEF}<40 \%$, creatinine clearance, and incomplete revascularization were independent predictors for 10 -year death/MI (all $p<0.01$ ) (Table 4).

\section{Discussion}

In this study, the long-term outcomes of patients after unprotected LM PCI were assessed in a large cohort of realworld patients in a large Chinese cardiovascular centre. The major findings of this study were as follows: (1) even with the use of a BMS or first-generation DES, PCI for unprotected LM disease showed favourable long-term results for up to 10 years; (2) compared with BMSs, DESs significantly reduced long-term adverse events, and IVUS-guided PCI was associated with a lower incidence of the composite death, stroke, or MI events; and (3) age and an LVEF $<40 \%$ are independent predictors for 10-year death, while age, LVEF $<40 \%$, creatinine clearance, and incomplete revascularization are independent predictors for 10-year death/ MI.

LM PCI outcomes after a 10-year follow-up duration are scarcely reported. Patients in the MAINCOMPARE (Revascularization for Unprotected Left Main Coronary Artery Stenosis: Comparison of Percutaneous Coronary Angioplasty Versus Surgical Revascularization) registry [25] who received BMSs for LM with less complex CAD showed a 10year survival probability of $83.1 \%$. In the LE MANS (Left Main Coronary Artery Stenting) registry, which included a wide spectrum of patients with CAD, as well as acute coronary syndromes, the 10-year survival after LM stenting was nearly $70 \%$ [11]. In the LE MANS prospective trial, which randomly evaluated LM stenting and CABG for unprotected LM stenosis with low and medium SYNTAX 
TABLE 2: Baseline lesion and procedure characteristics.

\begin{tabular}{|c|c|}
\hline & $N=913$ \\
\hline \multicolumn{2}{|l|}{ Coronary artery disease extent } \\
\hline Isolated LM & $8.0 \%(73)$ \\
\hline $\mathrm{LM}+1 \mathrm{VD}$ & $19.9 \%(182)$ \\
\hline $\mathrm{LM}+2 \mathrm{VD}$ & $35.9 \%(328)$ \\
\hline $\mathrm{LM}+3 \mathrm{VD}$ & $36.1 \%(330)$ \\
\hline Total occluded lesion & $4.4 \%(40)$ \\
\hline \multicolumn{2}{|l|}{ LM lesion location } \\
\hline Ostium & $11.4 \%(104)$ \\
\hline Shaft & $5.7 \%(52)$ \\
\hline Bifurcation & $82.9 \%(757)$ \\
\hline \multicolumn{2}{|l|}{ Main vessel } \\
\hline Reference vessel diameter, $\mathrm{mm}$ & $3.66 \pm 0.46$ \\
\hline Lesion length, $\mathrm{mm}$ & $20.7 \pm 15.3$ \\
\hline Diameter stenosis, \% & $83.0 \pm 10.7$ \\
\hline \multicolumn{2}{|l|}{ Side branch } \\
\hline Reference vessel diameter, $\mathrm{mm}$ & $2.96 \pm 0.38$ \\
\hline Lesion length, $\mathrm{mm}$ & $20.3 \pm 13.7$ \\
\hline Diameter stenosis, \% & $79.8 \pm 13.2$ \\
\hline \multicolumn{2}{|l|}{ Medina type for bifurcation lesion } \\
\hline $0,1,1$ & $4.1 \%(37)$ \\
\hline $1,0,0$ & $3.8 \%(29)$ \\
\hline $1,0,1$ & $7.9 \%(60)$ \\
\hline $1,1,0$ & $46.8 \%(354)$ \\
\hline $1,1,1$ & $36.6 \%(277)$ \\
\hline \multicolumn{2}{|l|}{ Lesion type } \\
\hline De novo & $96.4 \%(880)$ \\
\hline SYNTAX score & $24.5 \pm 7.4$ \\
\hline SYNTAX score $\leq 32$ & $86.3 \%(788)$ \\
\hline SYNTAX score $>32$ & $13.7 \%(125)$ \\
\hline \multicolumn{2}{|l|}{ Procedure access } \\
\hline Transradial approach & $45.7 \%(417)$ \\
\hline Transfemoral approach & $54.3 \%(496)$ \\
\hline \multicolumn{2}{|l|}{ Stent type } \\
\hline Bare metal stent & $12.0 \%(110)$ \\
\hline Drug-eluting stent & $88.0 \%(803)$ \\
\hline 1st generation drug-eluting stent & $15.0 \%(137)$ \\
\hline 2nd generation drug-eluting stent & $72.9 \%(666)$ \\
\hline Number of stents per patient & $2.15 \pm 1.18$ \\
\hline Total stent length, mm & $32.1 \pm 19.3$ \\
\hline 2 -stent strategy & $26.5 \%(242)$ \\
\hline Crush & $16.3 \%(149)$ \\
\hline T-stent & $3.3 \%(30)$ \\
\hline $\mathrm{V}$ or kissing stent & $5.4 \%(49)$ \\
\hline Culotte & $1.5 \%(14)$ \\
\hline Residual SYNTAX score & $4.86 \pm 6.26$ \\
\hline Guidance with IVUS & $39.5 \%(361)$ \\
\hline Performed by an experienced operator* & $83.2 \%(760)$ \\
\hline
\end{tabular}

* Experienced operator defined as those performed, at least, 15 LM PCIs per year for, at least, 3 consecutive years.

scores, the patients in the PCI arm reached nearly $80 \% 10$ year survival [26]. In the ASAN-MAIN (ASAN Medical Center-Left MAIN Revascularization) registry, the 10-year survival was $84.1 \%$ in patients with LM bare metal stenting [27]. In a recent report comparing provisional stenting vs. the two-stent strategy in patients with LM bifurcation lesions, the 10-year survival of the overall patients was over $70 \%$ [28]. To our knowledge, this study included the largest cohort of 913 LM PCI patients with 10-year follow-up results. In this study, the 10-year estimated rate of all-cause death was $14.9 \%$ with cardiac death accounting for only $7.9 \%$, which was even lower than the results of the abovementioned studies. Overall, the results of this study together with those of the others mentioned above suggest that the very long-term outcomes of PCI for LM were acceptable. Furthermore, a recent study demonstrated that LM PCI using DESs in those patients with high-risk features that represent exclusion criteria of previous randomized trials (e.g., AMI within 1 week, $\mathrm{LVEF}<30 \%$, and cardiogenic shock) achieved the same long-term outcomes compared with low-risk patients [29]. Our latest study analysed all PCI patients in 2013 in our centre, and during a 2-year follow-up period, we found that LM PCI was not an independent risk factor for any clinical adverse events [30]. Thus, it seems that interventionists should not consider LM lesions per se as a particular high-risk subgroup any more with contemporary PCI treatment. On the other hand, in this retrospective study, a large portion of the patients (21.6\%) evaluated for LM PCI was rejected by surgeons due to patient comorbidities or surgical ineligibility, and those patients were proven to have a worse long-term prognosis.

After the introduction of DESs, with a remarkable reduction in restenosis and repeat revascularization, LM PCI with DESs has been confirmed to have more favourable clinical outcomes than that with BMSs [6-11]. However, it could be associated with increased risk of very late stent thrombosis. Due to inaccuracy of angiography in LM coronary stenosis assessment [31], IVUS guidance has been proven to be more important than for non-LM lesions and improve the long-term prognosis in patients with unprotected LM CAD undergoing PCI [18, 32-34]. Our study had same findings: compared with BMSs, first-generation DESs significantly reduced long-term adverse events. The majority of definite/probable stent thrombosis was very late thrombosis, and it may be partly explained by the predominant use of DESs in this population; IVUS-guided PCI was associated with a lower incidence of the composite death, stroke, or MI events. This retrospective study revealed no difference in the long-term prognosis with the 1- or 2-stent strategy. Most patients with LM bifurcations received the 1-stent strategy, while the 2-stent strategy was mainly chosen for LM bifurcation lesions with more complex anatomy or true bifurcations and was performed by high-volume operators [19]. The techniques used in this series were similar to European Bifurcation Club recommendations [32]. In this observational study, DM status was not seen to be significantly associated with worse long-term adverse events, and this finding was inconsistent with previous reports [35-38].

This study demonstrated that age and an LVEF $<40 \%$ were independent predictors of 10-year death. On the other hand, age, $\mathrm{LVEF}<40 \%$, creatinine clearance, and incomplete revascularization were independent predictors for 10-year death and MI. This finding was consistent with our previous short-term (15-month follow-up) small cohort (220 LM PCI patients) study from 2003 to 2006 in our centre, which also found that an LVEF $<40 \%$ and incomplete revascularization (residual SS $\geq 8$ ) were independent predictors for death and 
TABLE 3: 10-year clinical outcomes.

\begin{tabular}{lcc}
\hline & Estimated event rates $N=913$ & $95 \%$ confidence interval \\
\hline All-cause death & $14.9 \%(136)$ & $12.5 \%-17.3 \%$ \\
Cardiac death & $7.9 \%(72)$ & $6.1 \%-9.7 \%$ \\
MI & $11.0 \%(100)$ & $8.9 \%-13.0 \%$ \\
Periprocedural MI* & $3.3 \%(30 / 913)$ & $3.0 \%-3.6 \%$ \\
Target-vessel-related MI & $9.7 \%(89)$ & $7.8 \%-11.7 \%$ \\
Stroke & $7.1 \%(65)$ & $5.4 \%-8.8 \%$ \\
Any revascularization & $25.0 \%(228)$ & $22.1 \%-27.8 \%$ \\
TVR & $16.1 \%(147)$ & $13.7 \%-18.5 \%$ \\
TLR & $9.9 \%(90)$ & $7.9 \%-11.8 \%$ \\
Definite/probable ST & $4.3 \%(39)$ & $2.9 \%-5.6 \%$ \\
Definite ST & $1.2 \%(11)$ & $0.4 \%-2.0 \%$ \\
Probable ST & $3.1 \%(28)$ & $1.9 \%-4.2 \%$ \\
Acute & $0.2 \%(2)$ & $0 \%-0.6 \%$ \\
Subacute & $0.4 \%(4)$ & $0 \%-0.9 \%$ \\
Late & $0.3 \%(3)$ & $0 \%-0.8 \%$ \\
Very late & $3.3 \%(30)$ & $2.1 \%-4.5 \%$ \\
Death + stroke + MI & $25.6 \%(234)$ & $22.7 \%-28.5 \%$ \\
Cardiac death + target-vessel MI + TLR & $23.1 \%(211)$ & $20.3 \%-25.9 \%$ \\
Death + MI + any revascularization & $41.9 \%(383)$ & $38.7 \%-45.2 \%$ \\
Death + MI + stroke + any revascularization & $45.9 \%(419)$ & $42.6 \%-49.2 \%$ \\
\hline
\end{tabular}

Values are reported as $\%(n)$; $*$ periprocedural MI was defined as a creatine kinase concentration $>2$ times the upper limit of normal within 48 hours after the procedure; $\mathrm{TVR}=$ target-vessel revascularization; $\mathrm{TLR}=$ target lesion revascularization; $\mathrm{ST}=$ stent thrombosis; and $\mathrm{MI}=$ myocardial infarction .

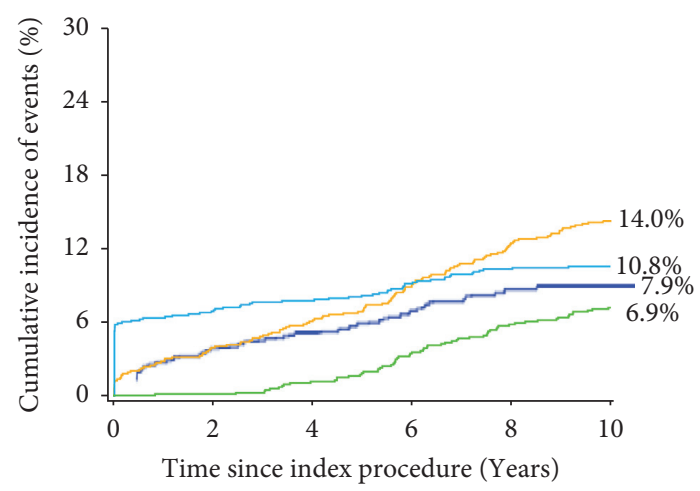

Number at risk:

\begin{tabular}{|c|c|c|c|c|c|c|}
\hline Death & 913 & 877 & 857 & 831 & 801 & \\
\hline MI & 913 & 847 & 840 & 827 & 816 & \\
\hline Stroke & 913 & 912 & 903 & 883 & 862 & \\
\hline $\begin{array}{l}\text { Cardiac } \\
\text { death }\end{array}$ & 913 & 877 & 857 & 831 & 801 & \\
\hline
\end{tabular}

(a)

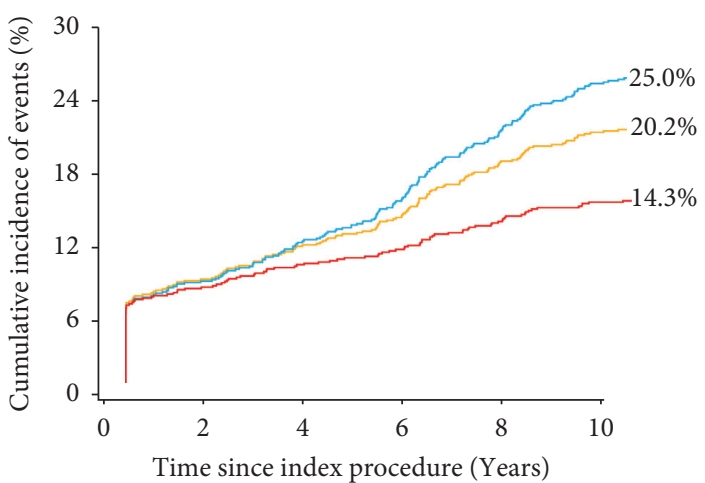

Number at risk:

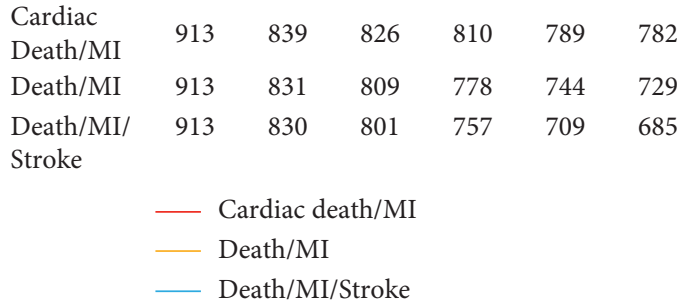

(b)

Figure 2: Long-term efficiency and safety after LM PCI. (a) Kaplan-Meier curves for death, cardiac death, MI, and stroke events; (b) Kaplan-Meier curves for composite events including death/MI and death/MI/stroke. MI = myocardial infarction.

MI after multivariate analysis [39]. These results were also consistent with two recently published long-term studies $[40,41]$.

Our study has potential clinical implications. It seems that interventionists should be more optimistic for the very long-term outcomes of LM PCI, but there still are some issues that need to be noted. First, these consecutive 916 LM
PCI cases from 2004 to 2008 only accounted for $4.67 \%$ of the total PCI cases at the same time; a large proportion of patients with LM disease were recommended to undergo CABG treatment. Second, just as our previous study demonstrated that operator experience affected prognosis after LM PCI [17], whether the conclusions of this study achieved in a large cardiac centre where most LM PCIs were 


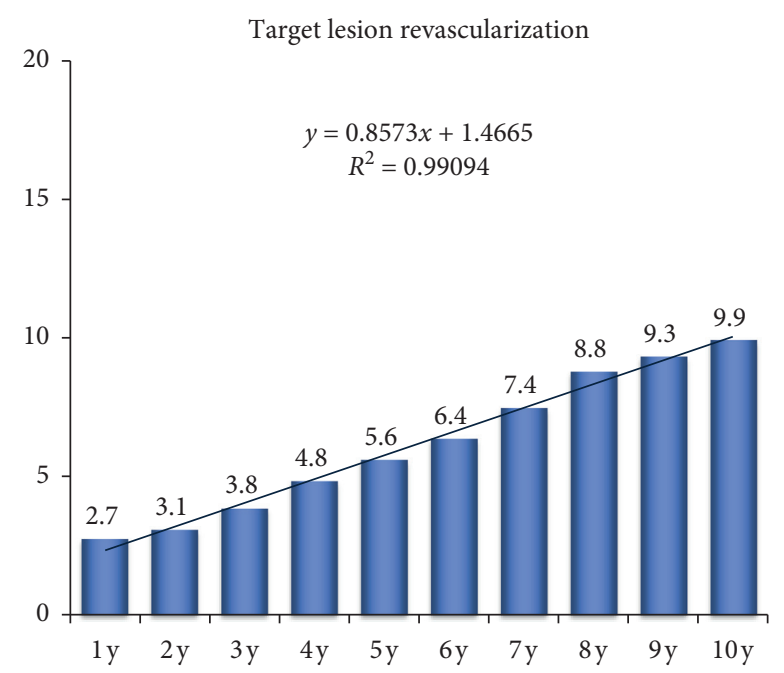

(a)

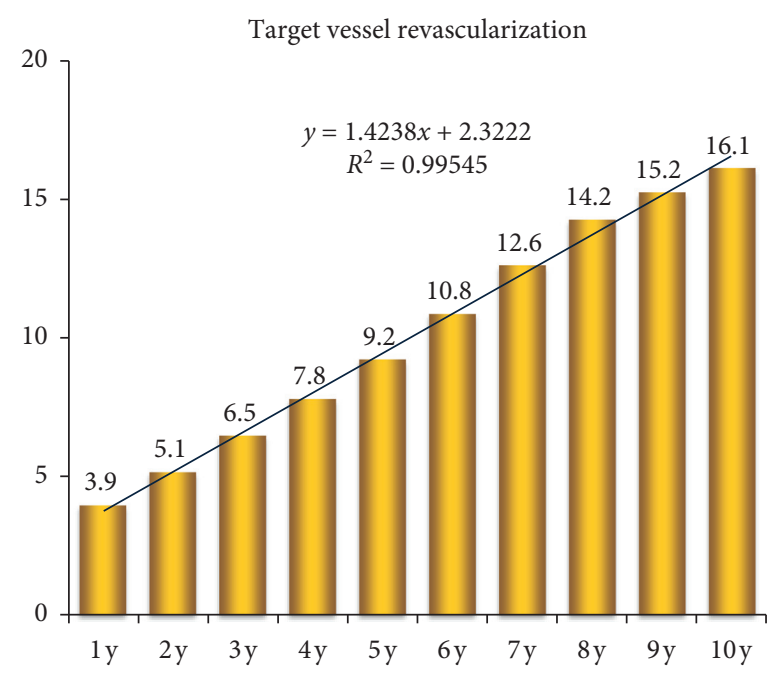

(b)

Figure 3: 10-year target lesion revascularization and target-vessel revascularization. Annual target lesion revascularization (a) and targetvessel revascularization (b) event rates.

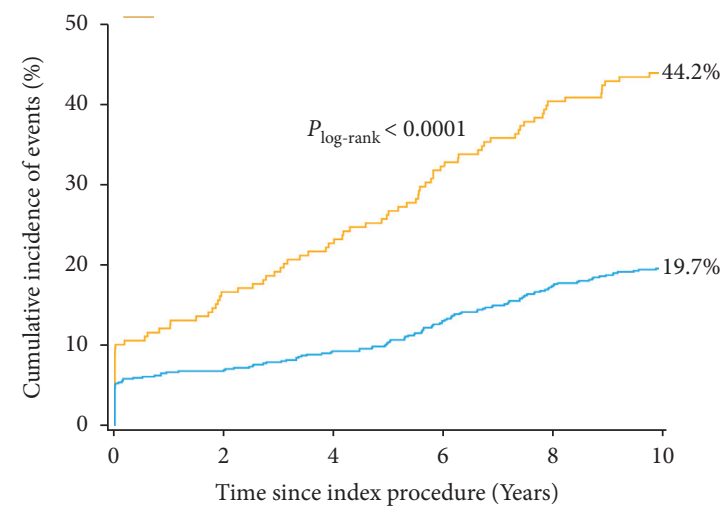

Number at risk:

$\begin{array}{lllllll}\begin{array}{l}\text { Surgical turn } \\ \text { down }\end{array} & 197 & 164 & 152 & 133 & 117 & 110 \\ \text { Regular } & 716 & 666 & 649 & 624 & 592 & 575\end{array}$

Surgical exclusion population

- Regular population

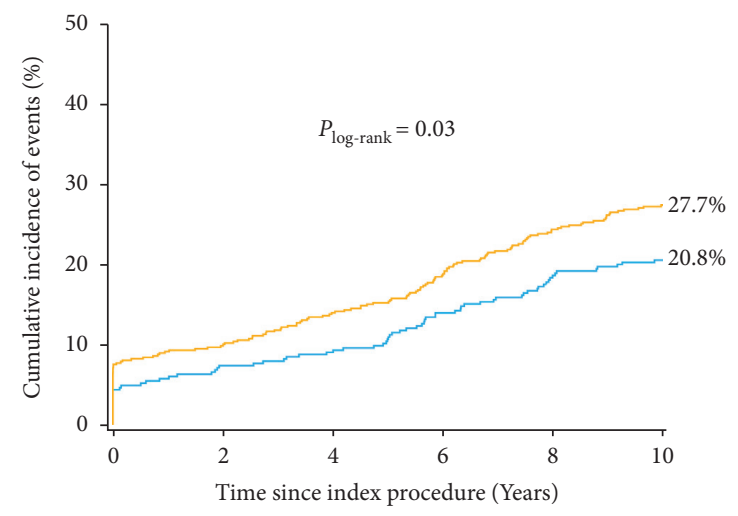

Number at risk:

$\begin{array}{llllll}\begin{array}{l}\text { Angiography- } \\ \text { guidance }\end{array} & 552 & 496 & 474 & 447 & 416\end{array}$

IVUS-

guidance

361

334

327

$310 \quad 293$

286

__ Angiography guidance

_ IVUS guidance

(a)

(b)

Figure 4: Continued. 


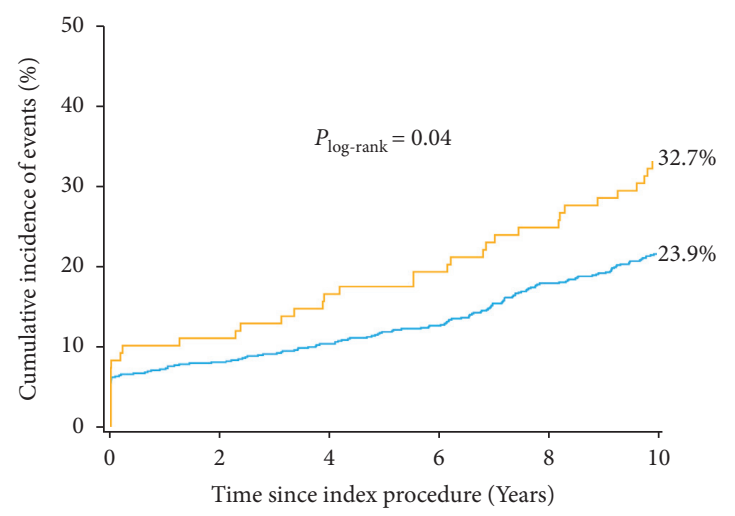

Number at risk:

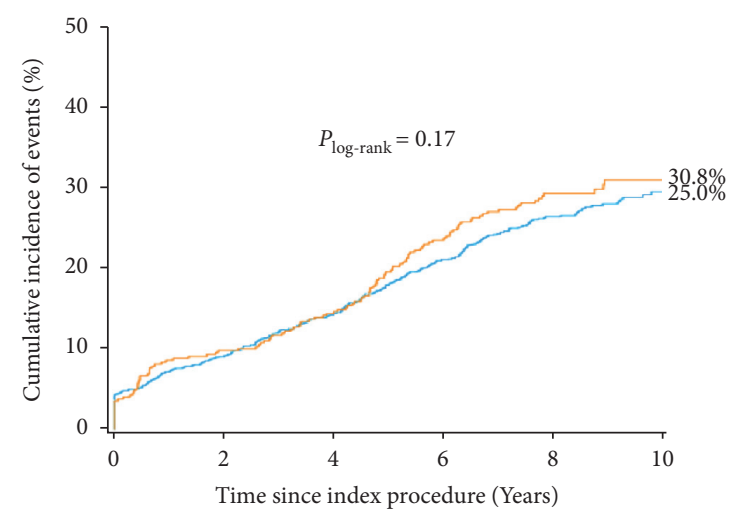

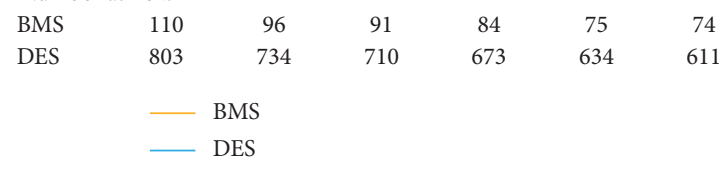

(c)

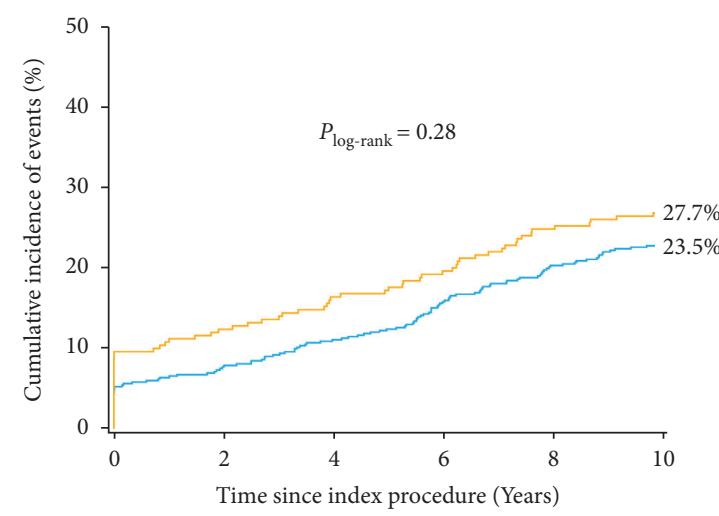

Number at risk:

$\begin{array}{lllllll}\text { Two-stent } & 242 & 211 & 201 & 194 & 180 & 175\end{array}$

$\begin{array}{lllllll}\text { One-stent } & 515 & 474 & 457 & 433 & 409 & 394\end{array}$

- Two stents

One stents
Number at risk:

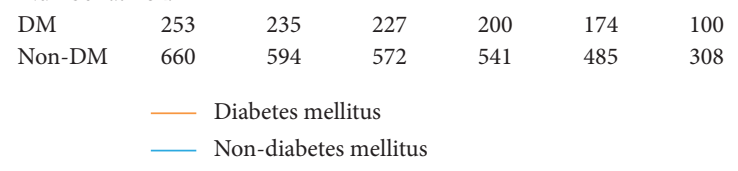

(d)

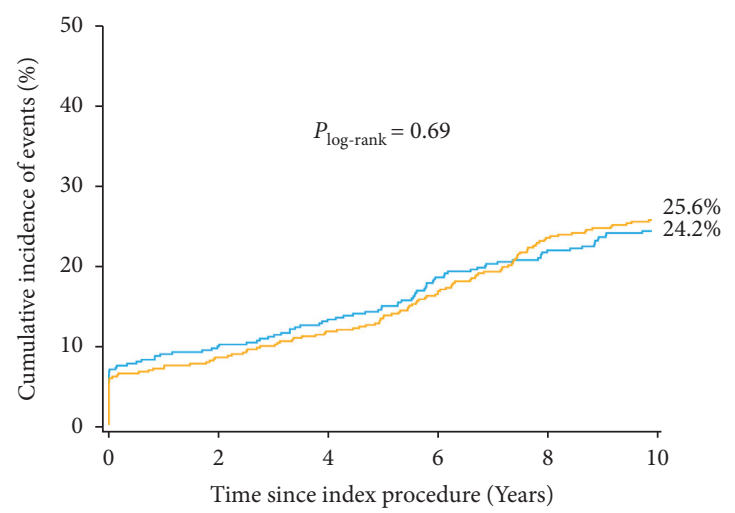

Number at risk:

$\begin{array}{lllllll}\text { Transradial } & 417 & 376 & 363 & 341 & 327 & 316\end{array}$

$\begin{array}{llllll}\text { Transfemoral } & 496 & 454 & 438 & 416 & 382\end{array}$

_ Transfemoral

_ Transradial

(e)

Figure 4: Continued. 


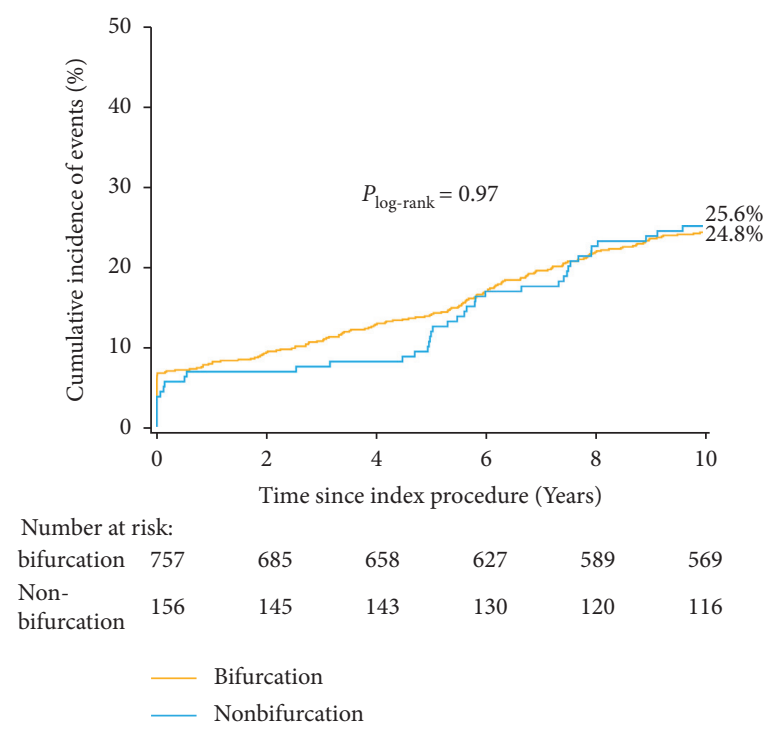

(g)

FIGURE 4: Survival curves of 10-year death/MI/stroke events among subgroups. The surgical exclusion population included patients meeting any one of the following criteria: chronic obstructive pulmonary disease, left ventricular ejection fraction $<35 \%$, age $>70$, acute myocardial infarction with haemodynamic instability, creatinine clearance $<50$, or bleeding history within 6 months. BMS $=$ bare metal stent; DES = drug-eluting stent; and IVUS = intravascular ultrasound. Other abbreviations are as in Figure 2.

TABLe 4: Predictors of long-term adverse events after LM PCI.

\begin{tabular}{lcc}
\hline & Hazard ratio $(95 \%$ confidence interval $)$ & $p$ value \\
\hline Death & & \\
Bifurcation lesion & $1.11(0.70,1.77)$ & 0.66 \\
EF $<40 \%$ & $4.51(1.98,10.28)$ & $<0.001$ \\
Incomplete revascularization & $1.06(0.73,1.55)$ & 0.76 \\
Diabetes & $0.98(0.67,1.44)$ & 0.92 \\
Age & $1.71(1.37,2.12)$ & $<0.001$ \\
CCr & $0.10(0.92,1.07)$ & 0.89 \\
\hline Death/MI & & 0.66 \\
Bifurcation lesion & $0.87(0.73,1.04)$ & 0.02 \\
EF $<40 \%$ & $1.89(1.09,3.28)$ & 0.03 \\
Incomplete revascularization & $1.16(1.01,1.33)$ & 0.49 \\
Diabetes & $1.05(0.98,1.22)$ & $<0.001$ \\
Age & $1.15(1.07,1.24)$ & 0.001 \\
CCr & $1.04(1.02,1.06)$ & \\
\hline
\end{tabular}

Incomplete revascularization was defined as a SYNTAX revascularization index $<100 \%$. Abbreviations are as in Tables 1 and 2.

performed by experienced operators [19] could be expanded to other centres needs to be further confirmed.

\section{Study Limitations}

This report has some limitations that should be acknowledged. First, the major limitation of this study is its observational design, which introduces latent, unrecognized, or unmeasured variables that could result in hidden bias. Second, this study only included patients who underwent LM PCI; therefore, the very long-term outcomes of LM PCI in comparison with those of LM-CABG cannot be evaluated by this study. Third, the study used a creatine kinase concentration $>2$ times to define periprocedural MI, which is an old definition that might overestimate MI rates. Finally, these data are from 2004 to 2008, which cannot reveal the latest advances in the PCI era. Including patients with now-historical stents, this report does not include physiologic assessment (FFR/iFR, etc) and has limited use of imaging. However, with utility of these modern assessment modalities, one would expect even more favourable results of PCI for LM disease in contemporary practice.

\section{Conclusions}

The current report, drawn from a large cohort of consecutive patients who underwent LM PCI, indicated that 
even with the use of BMSs or first-generation DESs, PCI for unprotected LM disease showed favourable long-term results for up to 10 years. In addition, age and the LVEF are key factors for long-term prognosis following LM PCI. Further study should focus on the long-term outcomes of LM PCI in comparison with LM-CABG to provide more evidence.

\section{Data Availability}

The clinical and procedural data used to support the findings of this study are included within the article.

\section{Ethical Approval}

Data collection for this study was approved by the institutional review board central committee at Fuwai Hospital and National Center for Cardiovascular Diseases of China.

\section{Consent}

All human patients provided written, informed consent.

\section{Conflicts of Interest}

The authors report no conflicts of interest in regards to this manuscript.

\section{Acknowledgments}

The authors are grateful to the Department of Cardiology, Cardiovascular Institute of Fu Wai Hospital, for its help in recruiting patients. The authors thank all members who contributed to the study. This work was supported by CAMS Innovation Fund for Medical Sciences (CIFMS) (2016-I2M1-009).

\section{Supplementary Materials}

The supplementary file includes the appendix of table with detailed description of all-cause death (Supplementary Materials)

\section{References}

[1] G. W. Stone, J. F. Sabik, P. W. Serruys et al., "Everolimus eluting stents or bypass surgery for left main coronary artery disease," New England Journal of Medicine, vol. 375, no. 23, pp. 2223-2235, 2016.

[2] T. Mäkikallio, N. R. Holm, M. Lindsay et al., "Percutaneous coronary angioplasty versus coronary artery bypass grafting in treatment of unprotected left main stenosis (NOBLE): a prospective, randomised, open-label, non-inferiority trial," The Lancet, vol. 388, no. 10061, pp. 2743-2752, 2016.

[3] S. Windecker, P. Kolh, F. Alfonso et al., "2014 ESC/EACTS guidelines on myocardial revascularization," Kardiologia Polska, vol. 72, pp. 1253-1379, 2014.

[4] S. D. Fihn, J. C. Blankenship, and K. P. Alexander, "ACC/ AHA/AATS/PCNA/SCAI/STS focused update of the guideline for the diagnosis and management of patients with stable ischemic heart disease: a report of the American college of cardiology/American heart association task force on practice guidelines, and the American association for thoracic surgery, preventive cardiovascular nurses association, society for cardiovascular angiography and interventions, and society of thoracic surgeons," Journal of the American College of Cardiology, vol. 64, pp. 1929-1949, 2014.

[5] F. Burzotta, J. F. Lassen, A. P. Banning et al., "Percutaneous coronary intervention in left main coronary artery disease: the 13th consensus document from the European Bifurcation Club," EuroIntervention, vol. 14, no. 1, pp. 112-120, 2018.

[6] S.-J. Lefèvre, Y.-H. Kim, B.-K. Lee et al., "Sirolimus-eluting stent implantation for unprotected left main coronary artery stenosis," Journal of the American College of Cardiology, vol. 45, no. 3, pp. 351-356, 2005.

[7] M. Valgimigi, C. A. G. van Mieghem, A. T. L. Ong et al., "Short- and long-term clinical outcome after drug-eluting stent implantation for the percutaneous treatment of left main coronary artery disease," Circulation, vol. 111, no. 11, pp. 1383-1389, 2005.

[8] A. Cheiffo, G. Stankovic, E. Bonizzoni et al., "Early and midterm results of drug-eluting stent implantation in unprotected left main," Circulation, vol. 111, no. 6, pp. 791-795, 2005.

[9] A. Erglis, I. Narbute, I. Kumsars et al., "A randomized comparison of paclitaxel-eluting stents versus bare-metal stents for treatment of unprotected left main coronary artery stenosis," Journal of the American College of Cardiology, vol. 50, no. 6, pp. 491-497, 2007.

[10] Y. H. Kim, D. W. Park, and S. W. Lee, "For the revascularization for unprotected left main coronary artery stenosis: comparison of percutaneous coronary angioplasty versus surgical revascularization investigators. Long-term safety and effectiveness of unprotected left main coronary stenting with drug-eluting stents compared with bare-metal stents," Circulation, vol. 120, pp. 400-407, 2009.

[11] P. E. Buszman, P. P. Buszman, R. S. Kiesz et al., "Early and long-term results of unprotected left main coronary artery stenting," Journal of the American College of Cardiology, vol. 54, no. 16, pp. 1500-1511, 2009.

[12] J. Mallidi, A. R. Atreya, J. Cook et al., "Long-term outcomes following fractional flow reserve-guided treatment of angiographically ambiguous left main coronary artery disease: a meta-analysis of prospective cohort studies," Catheterization and Cardiovascular Interventions, vol. 86, no. 1, pp. 12-18, 2015.

[13] W. F. Fearon, A. S. Yong, G. Lenders et al., "The impact of downstream coronary stenosis on fractional flow reserve assessment of intermediate left main coronary artery disease," JACC: Cardiovascular Interventions, vol. 8, no. 3, pp. 398-403, 2015.

[14] A. S. C. Yong, D. Daniels, B. De Bruyne et al., "Fractional flow reserve assessment of left main stenosis in the presence of downstream coronary stenoses," Circulation: Cardiovascular Interventions, vol. 6, no. 2, pp. 161-165, 2013.

[15] B. Modi, T. van de Hoef, J. Piek, and D. Perera, "Physiological assessment of left main coronary artery disease," EuroIntervention, vol. 13, no. 7, pp. 820-827, 2017.

[16] S.-J. Park, J.-M. Ahn, S.-J. Kang et al., "Intravascular ultrasound-derived minimal lumen area criteria for functionally significant left main coronary artery stenosis," JACC: Cardiovascular Interventions, vol. 7, no. 8, pp. 868-874, 2014.

[17] J. M. de la Torre Hernández, F. Hernández Hernandez, F. Alfonso et al., "Prospective application of pre-defined intravascular ultrasound criteria for assessment of intermediate left main coronary artery lesions," Journal of the American College of Cardiology, vol. 58, no. 4, pp. 351-358, 2011. 
[18] A. P. Banning, J. F. Lassen, F. Burzotta et al., "Percutaneous coronary intervention for obstructive bifurcation lesions: the 14th consensus document from the European Bifurcation Club," EuroIntervention, vol. 15, no. 1, pp. 90-98, 2019.

[19] B. Xu, B. Redfors, Y. Yang et al., "Impact of operator experience and volume on outcomes after left main coronary artery percutaneous coronary intervention," JACC: Cardiovascular Interventions, vol. 9, no. 20, pp. 2086-2093, 2016.

[20] M. Silvestri, P. Barragan, J. Sainsous et al., "Unprotected left main coronary artery stenting: immediate and medium- term outcomes of 140 elective procedures," Journal of the American College of Cardiology, vol. 35, no. 6, pp. 1543-1550, 2000.

[21] W. A. Tan, H. Tamai, S.-J. Park et al., "Long-term clinical outcomes after unprotected left main trunk percutaneous revascularization in 279 patients," Circulation, vol. 104, no. 14, pp. 1609-1614, 2001.

[22] A. Black, R. Cortina, I. Bossi, R. Choussat, J. Fajadet, and J. Marco, "Unprotected left main coronary artery stenting," Journal of the American College of Cardiology, vol. 37, no. 3, pp. 832-838, 2001.

[23] J. A. Bittl, Y. He, A. K. Jacobs, C. W. Yancy, and S.-L. T. Normand, "Bayesian methods affirm the use of percutaneous coronary intervention to improve survival in patients with unprotected left main coronary artery disease," Circulation, vol. 127, no. 22, pp. 2177-2185, 2013.

[24] G. W. Stone, A. P. Kappetein, and J. F. Sabik, "Five-year outcomes after PCI or CABG for left main coronary disease," New England Journal of Medicine, vol. 381, no. 19, pp. 1820-1830, 2019.

[25] K. B. Seung, D.-W. Park, Y.-H. Kim et al., "Stentsversus coronary-artery bypass grafting for left main coronary artery disease," New England Journal of Medicine, vol. 358, no. 17, pp. 1781-1792, 2008.

[26] P. E. Lee, P. P. Buszman, I. Banasiewicz-Szkróbka et al., "Left main stenting in comparison with surgical revascularization," JACC: Cardiovascular Interventions, vol. 9, no. 4, pp. 318-327, 2016.

[27] D. W. Park, Y. H. Kim, and S. C. Yun, "Long-term outcomes after stenting versus coronary artery bypass grafting for unprotected left main coronary artery disease: 10 -year results of bare-metal stents and 5-year results of drug-eluting stents from the ASAN-MAIN (ASAN Medical Center-Left MAIN Revascularization) Registry," Journal of the American College of Cardiology, vol. 56, no. 17, pp. 1366-1375, 2010.

[28] F. D’Ascenzo, M. Iannaccone, and F. Giordana, "Provisional vs. two-stent technique for unprotected left main coronary artery disease after ten years follow up: a propensity matched analysis," International Journal of Cardiology, vol. 211, pp. 37-42, 2016.

[29] Y. S. Oh, H. J. Lee, J. Lee et al., "Long-term clinical outcomes after a percutaneous coronary intervention with a drugeluting stent in patients with unprotected left main coronary artery disease excluded from clinical trials," Coronary Artery Disease, vol. 30, no. 4, pp. 239-248, 2019.

[30] L. Xu, Z. Gao, and Y. Song, "Impact of unprotected left main percutaneous coronary intervention on long-term clinical outcomes: a large single-center study," Coronary Artery Disease, vol. 30, no. 4, pp. 249-254, 2019.

[31] G. Toth, M. Hamilos, S. Pyxaras et al., "Evolving concepts of angiogram: fractional flow reserve discordances in 4000 coronary stenoses," European Heart Journal, vol. 35, no. 40, pp. 2831-2838, 2014.

[32] S.-J. Park, Y.-H. Kim, D.-W. Park et al., "Clinical impact of intravascular ultrasound guidance on long-term mortality in stenting for unprotected left main coronary artery stenosis," Circulation: Cardiovascular Interventions, vol. 2, no. 3, pp. 167-177, 2009.

[33] J. M. de la Torre Hernandez, J. A. Baz Alonso, J. A. Gómez Hospital et al., "Clinical impact of intravascular ultrasound guidance in drug-eluting stent implantation for unprotected left main coronary disease," JACC: Cardiovascular Interventions, vol. 7, no. 3, pp. 244-254, 2014.

[34] P. Andell, S. Karlsson, and M. A. Mohammad, "Intravascular ultrasound guidance is associated with better outcome in patients undergoing unprotected left main coronary artery stenting compared with angigarphy guidance alone," Circ Cardiovasc Interv, vol. 10, Article ID e004813, 2017.

[35] N. S. Bajaj, N. Patel, R. Kalra et al., "Percutaneous coronary intervention vs. coronary artery bypass grafting for left main revascularization: an updated meta-analysis," European Heart Journal-Quality of Care and Clinical Outcomes, vol. 3, no. 3, pp. 173-182, 2017.

[36] D. Giacoppo, R. Colleran, S. Cassese et al., "Percutaneous coronary intervention vs coronary artery bypass grafting in patients with left main coronary artery stenosis," JAMA Cardiology, vol. 2, no. 10, pp. 1079-1088, 2017.

[37] M. R. Khan, W. T. Kayani, W. Ahmad et al., "Analysis of comparison of 5-year outcomes of percutaneous coronary intervention versus coronary artery bypass grafting in patients with unprotected left main coronary artery in the era of drugeluting stents," The American Journal of Cardiology, vol. 120, no. 9, pp. 1514-1520, 2017.

[38] Y.-H. Kim, D.-W. Park, W.-J. Kim et al., "Validation of SYNTAX (Synergy between PCI with Taxus and Cardiac Surgery) score for prediction of outcomes after unprotected left main coronary revascularization," JACC: Cardiovascular Interventions, vol. 3, no. 6, pp. 612-623, 2010.

[39] R.-1. Gao, B. Xu, J.-1. Chen et al., "Immediate and long-term outcomes of drug-eluting stent implantation for unprotected left main coronary artery disease: comparison with bare-metal stent implantation," American Heart Journal, vol. 155, no. 3, pp. 553-561, 2008.

[40] D. J. F. M. Thuijs, A. P. Kappetein, and P. W. Serruys, "Percutaneous coronary intervention versus coronary artery bypass grafting in patients with three-vessel or left main coronary artery disease: 10-year follow-up of the multicentre randomised controlled SYNTAX trial," Lancet (London, England), vol. 394, no. 10206, pp. 1325-1334, 2019.

[41] S. Park, J.-M. Ahn, K. Lee et al., "Long-term (10-year) outcomes of stenting or bypass surgery for acute coronary syndromes and stable ischemic heart disease with unprotected left main coronary artery disease," American Heart Journal, vol. 218, pp. 9-19, 2019. 